\title{
Comparative scanning near-field optical microscopy studies of plasmonic nanoparticle concepts
}

\author{
Patrick Andrae*a, Paul Fumagalli ${ }^{\mathrm{b}}$, Martina Schmid ${ }^{\mathrm{a}, \mathrm{b}}$ \\ ${ }^{a}$ Helmholtz Zentrum Berlin, Nanooptical concepts for PV, Hahn-Meitner Platz 1, 14109 Berlin, \\ Germany; 'breie Universität Berlin, Department of Physics, Arnimallee 14, 14195 Berlin, Germany
}

\begin{abstract}
We use scanning near-field optical microscopy (SNOM) to characterize different plasmonic-nanoparticle situations with high spatial and spectral resolution in this comparative study. The near-field enhancement is measured with an aperture probe (Al coated glass fiber) and two CCD spectrometers for simultaneous detection of reflection and transmission. The images of transmission and reflection show a correlation to the topography. We present a new way to access the relative absorption and discuss the results with consideration of artifact influences. Near-field enhancements are deeper understood by imaging isolated particles. This near field will be compared to measurements of random-particle distributions. Therefore, we will show normalized reflection and transmission images of random structures that lay the foundation for an absolute interpretation of near-field images. The normalization considers both the far-field UV/VIS results and a reference image of the substrate. The near-field reflection of nanoparticle arrays shows an enhancement of $25 \%$. In view of specific applications, particle distributions implemented in two ways: as far-field scatters and as near field enhancing objects.
\end{abstract}

Keywords: scanning near-field optical microscopy, near field, illumination mode, nanoparticle distributions, plasmonics, near-field absorption, solar cells, aperture probe SNOM

\section{INTRODUCTION}

Scanning near-field optical microscopy with aperture illumination is one of the important techniques to obtain reliable data that combine the advantage of scanning-probe technology with optical microscopy [1-3]. The evanescent wave is directly interacting with the nanostructures and side effects like the tip interaction are less pronounced than in apertureless SNOM [4].

The interaction of light with particles is discussed a lot with far-field measurements and near-field simulations [5]. Regular nanoparticles and precisely assembled structures are shown in literature often with the link to metamaterials, i.e. chiral plasmons and plasmonic interaction in [6-8]. The effects are mostly investigated in the far field and discussed by looking at different wavelengths to compare spectral data with different alignments. The near field of the nanoparticles can be calculated by different simulation methods (i.e. Mie theory, finite-difference time-domain method, multiple multipole method) $[9,10]$. Yet, an intensive theoretical work has been devoted to compare calculated near-fields with the measured ones, which in fact show a sample-probe interaction superimposed [3]. This interaction will always arise in experimental investigations. They appear using apertureless or aperture scanning near-field optical microscopy. For specific research task, the large number of setups are versatile [11, 12].

To get a real image or rather a real understanding of particle-particle or particle-substrate interaction, deeper spectroscopic investigations are needed. For an application like using particles in solar cells to improve the absorption the interest is shifted from a basic understanding to an application focused investigation considering a relative comparison of near-fields [13]. To take it even further: there is a need of ensuring the best quality and the process stability for serial productions of nanosystems. Using a homebuilt aperture scanning near-field optical microscope has great benefits: high spectral resolution, change of light sources, simultaneous multi detection on different position and variety of samples.

In this paper, we will discuss the near-field effects of silver nanoparticles going from one particle to a random structure that can be prepared easily. However, one should keep in mind that the near field is influenced by the nanoparticle shape, distribution and material [13]. In addition, the substrate has an impact on the field distribution. The detection and the scanning near-field optical microscope can be used in different ways and setups. In our case, the SNOM is working in

Optical Micro- and Nanometrology V, edited by Christophe Gorecki, Anand Krishna Asundi,

Wolfgang Osten, Proc. of SPIE Vol. 9132, 91320F · (c) 2014 SPIE

CCC code: $0277-786 X / 14 / \$ 18 \cdot$ doi: $10.1117 / 12.2051760$

Proc. of SPIE Vol. 9132 91320F-1 
the illumination mode because of the simultaneous detection of reflection and transmission in fixed positions. This focus will bring us to the question: what conclusion can be drawn by simultaneous studies of the near field and how can they be compared to each other.

*patrick.andrae@helmholtz-berlin.de; +4930806243721; www.helmholtz-berlin.de/forschung/oe/enma/nanooptix/index_en.html

\section{THEORY}

The electromagnetic field of an illuminated object contains both propagating and evanescent parts [14]. In the far-field detection, the dipole with the dipole moment $p(t)$ oscillates in the $\mathrm{z}$ direction at one point $(\theta, \psi, R)$. The field can be described using (1), (2) and (3).

$$
\begin{gathered}
E_{R}=2\left(\frac{p(t)}{R^{3}}+\frac{p(t)}{c R^{2}}\right) \cos \theta \\
E_{\theta}=\left(\frac{p(t)}{R^{3}}+\frac{p(t)}{c R^{2}}+\frac{p(t)}{c^{2} R}\right) \sin \theta \\
H_{\psi}=\left(\frac{p(t)}{c R^{2}}+\frac{p(t)}{c^{2} R}\right) \sin \theta
\end{gathered}
$$

where $p(t)=p_{0} \cos \omega\left(t-\frac{R}{c}\right)$ and $R$ is the distance from the dipole's decelerated oscillation to the point where the field is evaluated. The terms $R^{2}$ and $R^{3}$ are dominating in the near field, while in the far-field region the terms are negligible. This is why the near field has a decay following power law and without a nano-collector, it does not emit energy. If an object is illuminated with light, the coherently oscillating dipoles will generate a field with structural information [15]. However, the detection of this event in the far field is not possible. For this reason, a probe must interact with the evanescent field and start to create a propagating field that can be detected. This probe is moved over the sample and gives a high resolution of optical and topography data. In our set up, the probe will act as a near-field source and the sample takes over the role of the nano- collector.

The interpretation of SNOM images is the main challenge [16-18]. Artifacts of different origin influence the intensity distribution: All this has to be considered in the discussion of the results. The comparison between two experimental situations shows the same artifacts if the samples are measured with the same setup and under the same conditions, while the comparison of simulation and experiments needs a big effort in data correction. The following list gives a short overview where contrast could come from: proximity fields, evanescent fields, standing waves, inhomogeneous illumination, confocal detection and topographic influences [16].

\subsection{Near-field studies of nanostructures}

The simulation of the near field with nanostructures is well known [19-22]. Many simulations on different particles were done and the near field was measured with diverse techniques. The evanescent wave at the tip of an aperture SNOM used in illumination mode is locally scattered by small objects and emitted as a propagating wave. This wave is detected. The finite skin depth and the manufacturing limit of the aperture give the limits for topography and optical resolution [23]. It is essential: the lower the size of the opening the higher the losses in the aperture. The transmission in a standard probe drops from $10^{-3}$ to $2 \cdot 10^{-12}$ as the diameter is reduced by a factor of five [24].

Simulations are concentrating on single-particle situations or interaction of homogenous structures. The interaction of many-particle arrays are important for applications [25]. Particles can have a stand-alone near field or interact in groups. If there is a defined space between the particles, they can couple like in enhanced waveguide-mode structures [26-28]. If the space is larger than $200 \mathrm{~nm}$ no enhancement will be observed and the near-field on the bare substrate is much lower than close to a plasmonic structure. 


\subsection{Artifacts}

The homebuilt SNOM set up works with a commercial straight fiber in a constant gap-scanning mode. It has been shown that with a standard bi-layer model (sample / air) the artifacts can be induced with the height movement of the tip to different $\mathrm{Z}$ positions [17]. This movement occurring in the constant amplitude mode is transferable to topography influences. The movement in detection planes will imply an effect on the optical image. In figure 1 the situation of varying tip height is shown.

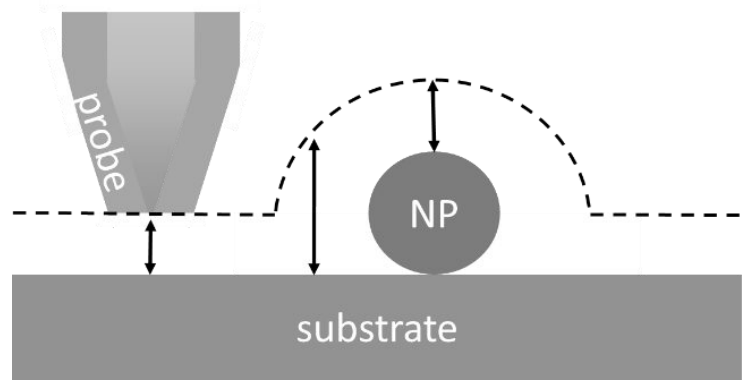

Figure 1. Probe movement (dashed line) over a silver nanoparticle (NP) on a glass substrate with different $\mathrm{z}$ positions (arrows).

The sample-probe distance is strongly related to both the tip diameter and the fabrication of the probe. The different $\mathrm{z}$ planes will give rise to an artifact at the surrounding of the nanoparticle and have to be considered in the interpretation of SNOM data. This effect can be seen in figure 1 that shows the varying sample-probe distance as a dashed line. We can use the intensity of the evanescent field at the point of height $Z$ above the surface of the sample in the way [17]:

$$
I(Z)=I(0) \exp \left(-Z \frac{4 \pi}{\lambda}\left(n_{s}{ }^{2} \sin ^{2} \bar{\theta}-1\right)^{\frac{1}{2}}\right)
$$

where $\lambda$ is the wavelength, $n_{s}$ the reflective index of the substrate and $\theta$ the angle formed between the surface and the tip. Furthermore, changes in the x position of the probe arise when working in shear force mode with an amplitude of 5 $10 \AA$. We will not dwell on these latter artifacts of $x$ variations because the probe diameter is many times higher than the movement.

\section{COMBINED INTERPRETATION OF TRANSMISSION AND REFLECTION}

The near-field absorption is a combination of reduced transmission and reflection, which cannot be separated in the near field easily. We will give a first idea how the combined interpretation of transmission and reflection can be obtained with several reference scans that has to be discussed in further works. To provide a clear absorption picture it is important that the energy of the incoming light is stable and shows a homogenous spectrum. If this is not the case, i.e. with LED or Xenon illumination sources, it is necessary to have a reference spectrum.

The classical far-field absorption is calculated with the formula (5),

$$
A_{\text {total }}=100 \%-\sum T-\sum R
$$

where the total absorption $A$ is calculated by subtracting transmission $T$ and reflection $R$ from a standard Reference corresponding to $100 \%$. This approach is not applicable to the near field. We prefer giving a two reference measuring process to calculate the interlinked image (7) of near-field transmission/reflection:

$$
\begin{gathered}
\text { refection }_{\text {nom }}(x, \lambda)=\frac{I_{\text {sample }}(x, \lambda) \cdot I_{\text {substrate_C }(x, \lambda)}}{I_{\text {substrate }}(x, \lambda) \cdot I_{\text {sample_C }_{\text {C }}(x, \lambda)}} \\
\text { relation }_{\text {sample }}(\mathrm{x}, \lambda)=\text { reflection }_{\text {nom }}(x, \lambda) \cdot R_{\text {far }}(\lambda)+\text { transmission }_{\text {nom }}(x, \lambda) \cdot T_{\text {far }}(\lambda)
\end{gathered}
$$


In (6) the normalisation of the sample's reflection or transmission intensity $I_{\text {sample }}$ is done with the near-field measurement of the pure substrate reflection or transmission intensity $I_{\text {substrate }}$ (including the intensity of the isolated probe $I_{\text {probe }}$ ). The whole term is then normalised with the fluctuation of the intensity of the source $I_{X_{-} C}$ that is measured for every point in the image. In (7) we calculate the linked transmission and reflection relation sample $(\mathrm{x}, \lambda)$ with respect to the normal transmission $T_{f a r}$ and reflection $R_{f a r}$ in the far field taken at the wavelength of the LASER. Using relation $_{\text {sample }}(\mathrm{x}, \lambda)$ it is possible to compare different particle and structure situations and to discuss absolute enhancement values.

\section{EXPERIMENTAL}

\subsection{Particle preparation}

We investigated particles formed by the thermal annealing of metal films [29]. Therefore a thin metal film was deposited by e-beam evaporation $(0.8 \AA \mathrm{A} / \mathrm{s}, 7.5 \mathrm{e}-6 \mathrm{mbar})$ and thermal evaporation $(1.2 \AA \mathrm{A} / \mathrm{s}, 6.2 \mathrm{e}-6 \mathrm{mbar})$, respectively. The film thickness of silver was chosen between 10 and $50 \mathrm{~nm}$ and annealing took place in a furnace at ambient atmosphere at temperatures between 300 and $500{ }^{\circ} \mathrm{C}$ for 10 up to $60 \mathrm{~min}$. All samples were characterized with AFM and SEM before the SNOM measurements. To focus on the main plasmon resonance the particle preparation was tuned with UV/VIS measurements to a particle resonance frequency of $532 \mathrm{~nm}$.
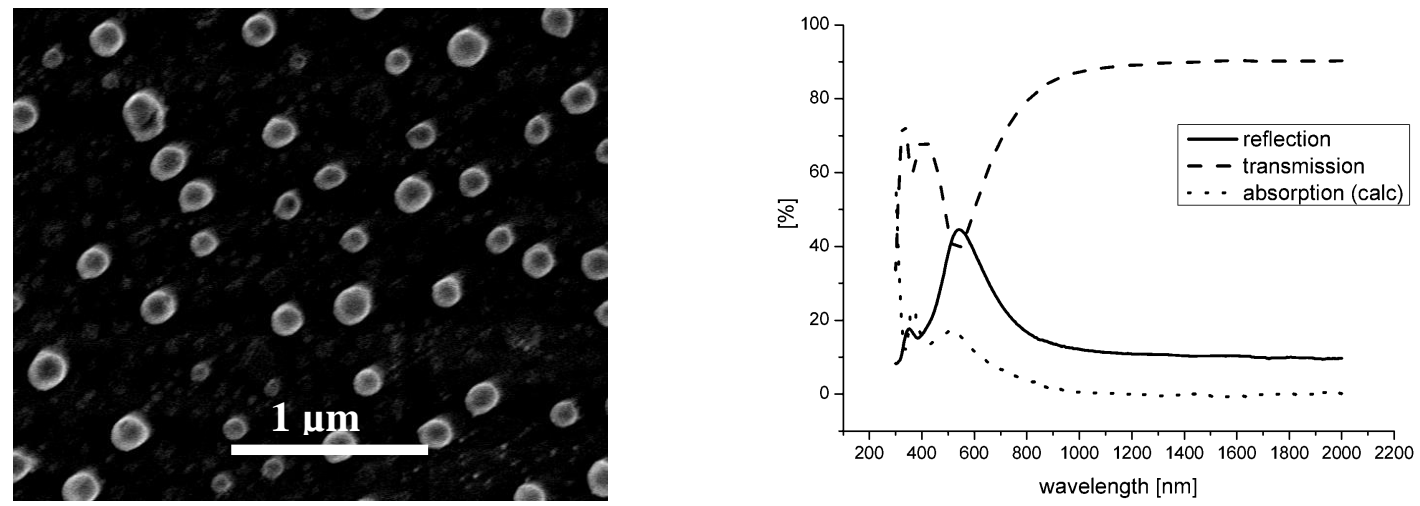

Figure 2. SEM picture of silver particles on a glass substrate with the associated UV/VIS Spectrum showing reflection (solid), transmission (dashed) and absorption calculated with (5) (dotted)

In figure 2, the SEM picture of the sample with the related UV/VIS spectrum is presented. The particles have a roundness factor from 0.8 to 0.95 and an average diameter of $200 \mathrm{~nm}$. The nearest-neighbor distance is around $100 \mathrm{~nm}$. For this sample, the main plasmon resonance is at $544 \mathrm{~nm}$ and a far-field / total absorption smaller than $20 \%$ is obtained. The substrate has a reflectivity of 0.08 and has areas with and without particles. Thus the sample has an integrated glass reference.

\subsection{Scanning near-field microscopy}

Classical SNOM technology can be found in many applications and analytical processes. The field is covered from optomagnetics up to Raman spectroscopy or two-color fluorescence imaging [30-32]. Commercial SNOM setups show limitations for the space and the detector one can use [3] and the number of light sources is limited. For this reason, here a homebuilt aperture scanning near-field optical microscope with commercial probes is used to investigate nanoparticles on glass substrates. The scheme of the setup is shown in Figure 3. The light source is selected by a fiber splice from a $532 \mathrm{~nm}$ LASER with10 mW. This light couples into an Al coated HSPC10 (Lovalite, multimode Fiber, $0.1 \mathrm{NA}, 10 \mu \mathrm{m}$ core) fiber probe with an aperture of $100 \mathrm{~nm}$ that is glued at the tuning fork. The distance control is done by a shear force mode with an amplitude of less than approximately $1 \mathrm{~nm}$. The sample is moved by an (x,y,z)-stage and due to the transparent substrate the reflection (2) and the transmission (3) can be measured with two CCD spectrometers in the range of $520 \mathrm{~nm}$ to $550 \mathrm{~nm}$. To characterize the light source in front of the fiber coupling the light is analyzed by a third 
spectrometer (1). The short-term drift of the laser induced by the thermal movement of optical parts and the long-term stability of the laser intensity are less than $7 \%$.

The sample is scanned in contact mode with different forces. A big sample scan of $10 \mu \mathrm{m}$ x $10 \mu \mathrm{m}$ is done first, followed by detailed scans and optical variations. A lens collects the far-field light. The integration time of the CCD spectrometers is set to $5 \mathrm{~ms}$ per pixel and the line scan is done with $40.000 \mathrm{~Hz}$. The standard image size is $50 \mathrm{px}$ squared. The sample is clamped with a specific holder in a vertical position. It can be changed without a repositioning of the optical path.

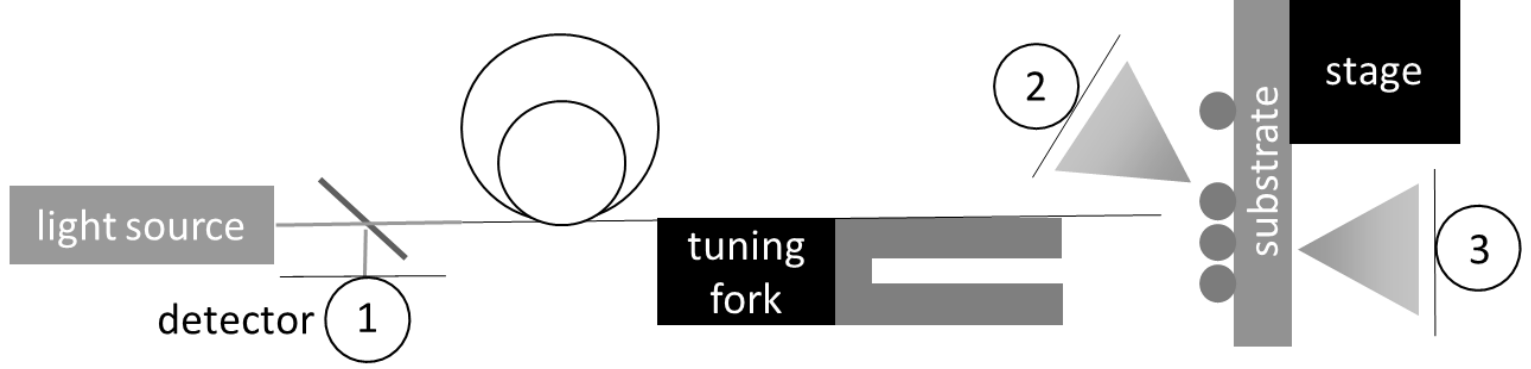

Figure 3. Layout of the SNOM with light coupling through a glass fiber on a tuning fork for illumination and three detectors: 1 - detector for incoming light intensity, 2 - detector for near-field reflection, 3 - detector for near-field transmission.

\section{NEAR-FIELD SINGNAL OF NANOSYSTEMS}

With the SNOM set up, we obtain four experimental images: topography, near-field reflection, near-field transmission and incoming light intensity. This paragraph shows standard topography and near-field pictures without any corrections or calculation. The optical data is without a baseline correction or other standard basis operations. The topography data is leveled and line corrected. The maximum size is $10 \mu \mathrm{m}$ by $10 \mu \mathrm{m}$ with a step size of $200 \mathrm{~nm}$. All measurements presented in one line are recorded at the same time. The size and resolution of all images depend on the amount of pixels and the step size of one pixel.

\subsection{Results of near-field investigation on an isolated particle}

We define isolated nanoparticles as particles with an outer boarder to outer boarder distance greater than the diameter. The spherical silver nanoparticles with a diameter of $175 \mathrm{~nm}$ are on a glass substrate. In Figure 4, a particle is imaged that shows a week near-field enhancement. For particles with a square shape, strong near-field enhancement would be expected [29]. No reflection at the particle position can be measured while there is a high transmission value.
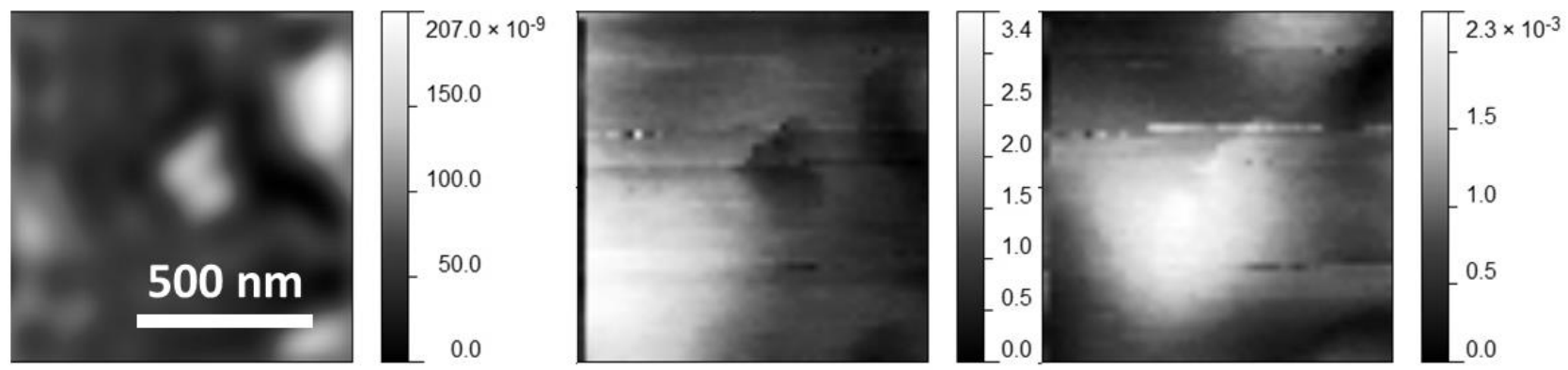

Figure 4 Isolated particle with topography, near-field reflection and near-field transmission (from left to right), scan step $30 \mathrm{~nm}$, overall size $1 \mu \mathrm{m} \times 1 \mu \mathrm{m}$.

In these images, the topography artifact can be seen articulately. The particle shows a very dark spot in the reflection image. If the probe height is changing due to the particles topography, the intensity of the reflection will decrease. The effect cannot be detected in the transmission image. 


\subsection{Random particle arrays}

Figure 5 illustrates the near-field measurements of random silver nanoparticles together with a topography image. The light comes from the front where the reflection image is placed. The lines indicate specific positions of the system that will be discussed. Same positions in different images are indicated by dots and lines.

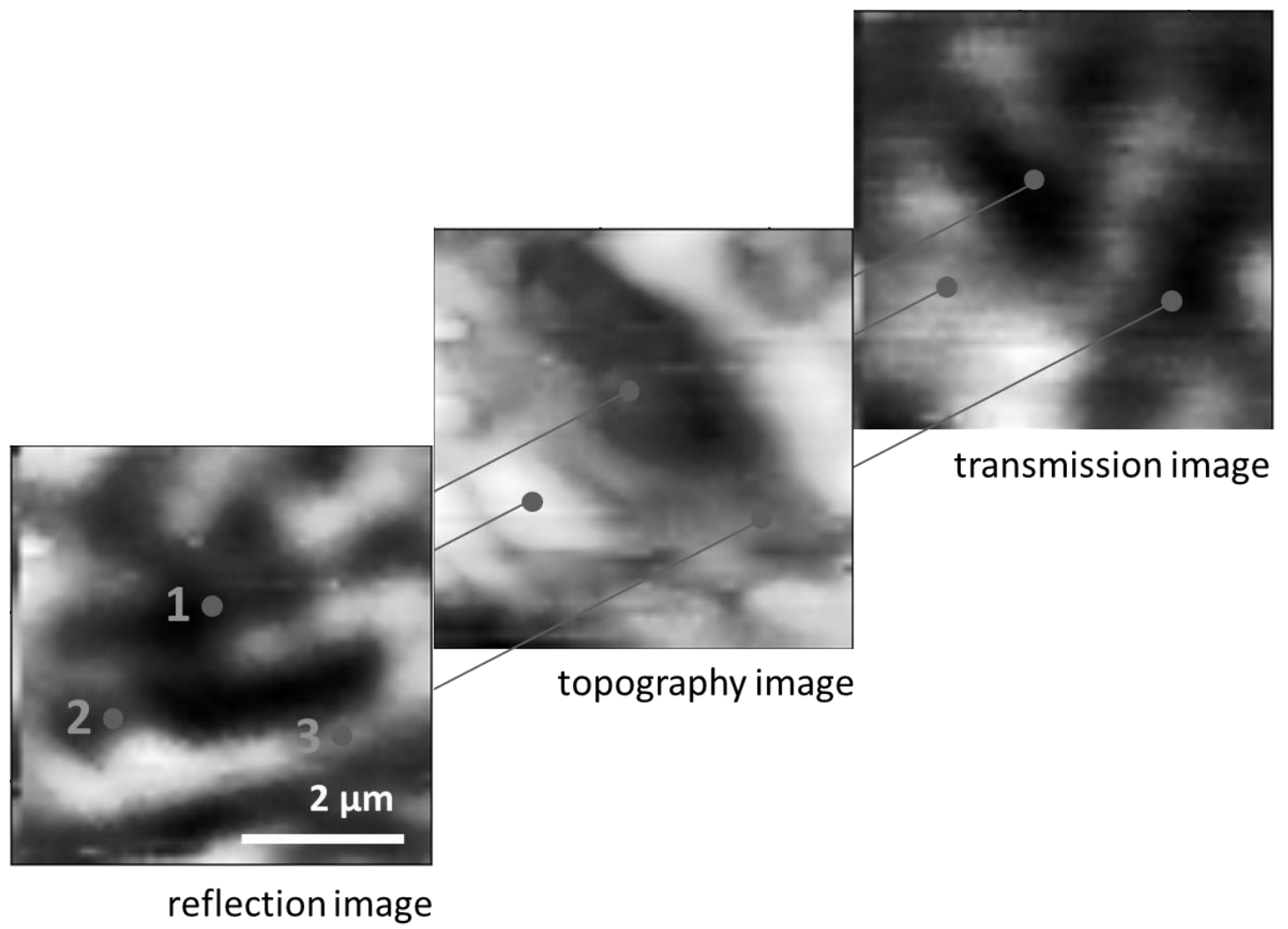

Figure 5. SNOM images of the sample with silver particles on top of a glass substrate. Line connected points indicate same positions in different images. Scan step size $100 \mathrm{~nm}$ on $5 \mu \mathrm{m}$ x $5 \mu \mathrm{m}$. The brightest color is related to high topography and high intensity, respectively.

Position 1 is next to a silver structure. Here no significant transmission and reflection can be detected. This was shown in a former publication [13]. The surface between particles does not show a high transmission like expected. Two effects can be discussed: the enhancement from particle plasmons is much higher than the total light going through a pure substrate and a higher parasitic absorption is expected from the particle, because compared to other particles the diameter is small. If these areas would be measured without any particles, they would show a value of the glass, this fact we use for the normalization of the near-field intensities. In position 2, the near-field next to the particle is enhanced like it would be expected from simulations [25]. The particles show a strong transmission and reflection at this point and may interact with neighboring particles. In position 3 , the particles seem to interact with each other and a higher reflection than transmission can be seen at a place where no particle is present. Figure 6 illustrate a more general case of plasmonic metal nanoparticles. We can observe the same effects as shown before. The central particle shows a strong field enhancement around its boundary. 

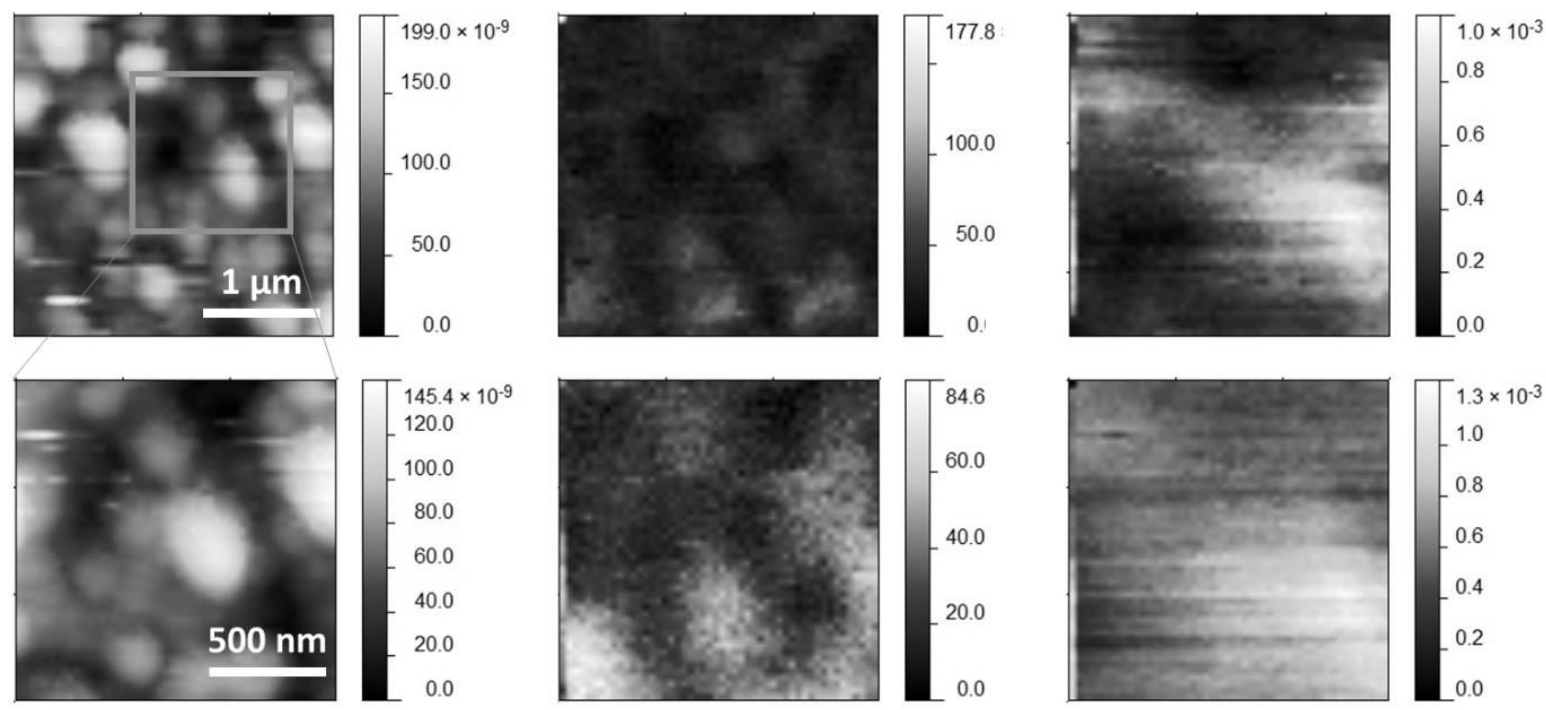

Figure 6. SNOM images of a dense particle configuration in topography, reflection and transmission of the near field with different resolutions. The structure height is around $200 \mathrm{~nm}$ with an equal diameter. The second row is a magnification of the first row (black square).

In areas that are particle free there is no field enhancement, if there is no particle interaction. In the lower row of images of figure 6 the two big particles on the right have a strong near-field effect. Especially within the transmission data, the near field is overlaid by far-field signal.

\section{NORMALISATION OF NEAR-FIELD SIGNALS}

Every data of transmission and reflection of the near field is detected together with the incoming light intensity. This intensity is coupled into a glass fiber and a change in intensity will have an effect on the near-field image contrast. As discussed before the transmission of the glass-fiber probe is $10 \mathrm{e}-3$ and the pure intensity will give a noise. This noise is stable in short term and varies by around $7 \%$. In more than 30 minutes the overall intensity is often increasing or decreasing constantly. In Figure 7 the taken reflection image (left) is divided by the intensity of the incoming light at every datapoint and results in the corrected middle image.
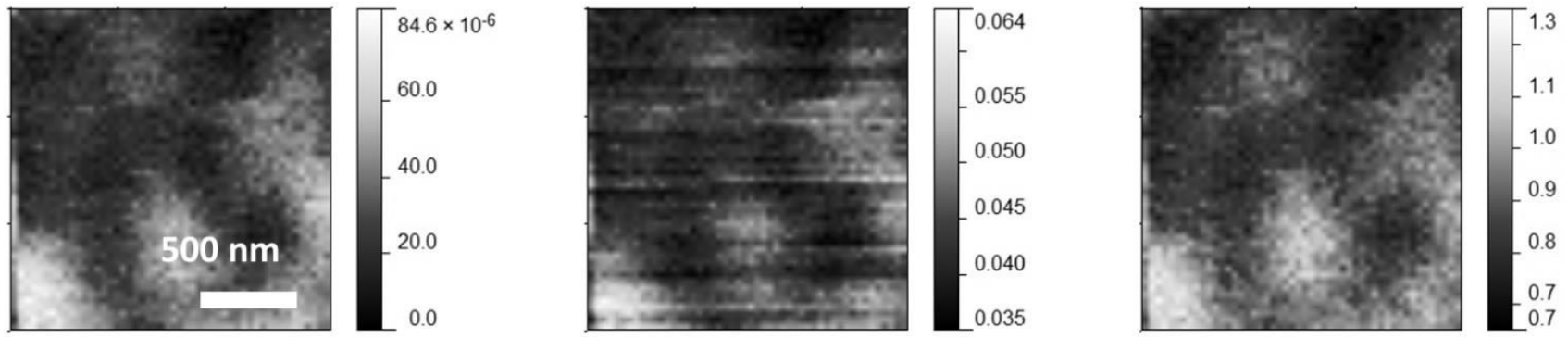

Figure 7. Reflection data (left) corrected with the intensity of the incoming laser light (middle) and corrected with the reference of the glass substrate (right), respectively. The scale is now related to a glass reference.

Both pictures should show the same intensity distribution, while the uncorrected picture has less noise. However, the reflected structures are smaller correlated to the topography and show a better spatial resolution. The bright spot in the lower left is mainly a result of a high intensity of the incoming light. The scan is done in horizontal line wise. Changes in the intensity are strongly related to the jump of the detection in the next line. The absolute intensity of the uncorrected data is normalized to compare with the scale of a glass-slide near-field intensity. The corrected reflection, shown in figure 7 (right), is related to a glass reference. The scale bar is shifted from an unusable scale to an intensity bar that can be compared with pure glass. If the value in the image shows 1.0, the reflection of the actual particle situation is equal to the reflection of the glass. Lower values indicate a decreased near field that can give a clue to a possible absorption. The correction is done for transmission and reflection in Figure 8 and we obtain intensities that are more meaningful. Both 
images are multiplied by the far-field values of transmission and reflection, as discussed before, and added in one remaining image that can show, where strong enhancement or reductions occurred. In Figure 8, the intensities are summed up following the equations (6) and (7).

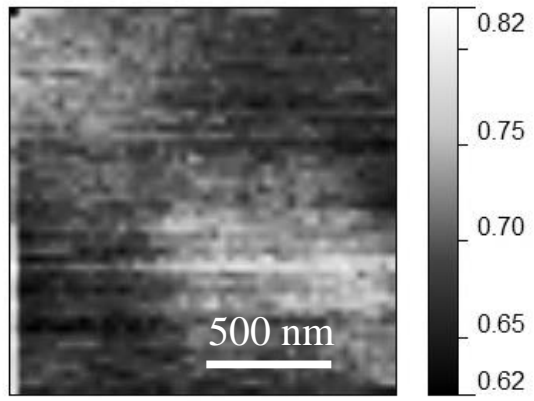

Figure 8. Sum of reflection and transmission normalized with the incoming light and the pure signal from a silver-free glass substrate.

The particles show a strong absorption in the whole image with the exception of the lower right part, where the transmission was very high. For the sum less than 1.0 the enhancement is lower than it was with pure glass. No particle will create a higher field. The reason of the lower values are related to the low transmission values $(<0.8)$ that are not shown here with an influence far-field factor of 0.92 according equation (7). For this reason, the calculation of the absorption and the reference measurement have to be improved and other particle distribution have to be measured in further publications. The standard reference will not fulfill the optimal calibration if only transmission can be calibrated. To get the values for $100 \%$ reflection another reference sample has to be used.

\section{CONCLUSION}

In summary, we have presented that random-nanoparticle structures with a resolution in the range of $100 \mathrm{~nm}$ can be detected with scanning near-field optical microscopy. The stability of the system is proved by repeatable images and reference scans of the substrate and the probe. The near field was measured in transmission and reflection and was correlated to the topography. A new method for a consistent evaluation of reference scans of the probe, incoming light intensity and the substrate near field was presented. The single-particle artifacts overlapped the images and it is a challenge to overcome the optical resolution limit of the aperture probe. Yet, we were able to show for random silver particle arrays that a correction of the incoming light creates both a sharper image of the reflection and scans can be compared to the topography. We showed that the near-field intensity becomes more meaningful if it is normalized to a reference without near-field enhancement. The particle array we presented has no overall enhancement in transmission and reflection. Yet, the enhancement for the reflection is higher than $100 \%$ compared to a pure substrate.

\section{ACKNOWLEDGEMENTS}

Regarding the SNOM setup, the collaboration with all members and technicians of the Fumagalli group from the Freie Universität Berlin as well as Phillip Manley of the Nanooptix Group from the HZB for all near-field discussion is acknowledged. Funding from the Helmholtz-Association for Young Investigator groups within the Initiative and Networking fund (VH-NG-928) is greatly acknowledged. This work was supported in part by the Stiftung der Deutschen Wirtschaft (sdw) gGmbH. 


\section{REFERENCES}

[1] Dürig, U., Pohl, D.W. and Rohner, F., "Near-field optical-scanning microscopy, J. Appl. Phys. 59(10), 3318-3327 (1986).

[2] Kirstein, S., "Scanning near-field optical microscopy", Current Opinion in Colloid \& Interface Science 4, 256 264 (1999).

[3] Hecht, B., Sick, B., Wild, U. P., Deckert,, V., Zenobi, R., Martin, O. J.F. and Pohl, D. W., "Scanning near-field optical microscopy with aperture probes: Fundamentals and applications", J. Chem. Phys. 112, 7761 (2000).

[4] Buil, S., Aubineau, J., Laverdant, J. and Quelin, X., "Local field intensity enhancements on gold semicontinuous films investigated with an aperture nearfield optical microscope in collection mode", J. Appl. Phys. 100, 063530 (2006).

[5] Kelly, L. K., Coronado, E., Zhao L. L. and Schatz, G. C., "The Optical Properties of Metal Nanoparticles: The Influence of Size, Shape, and Dielectric Environment", J. Phys. Chem. B. 107, 668-677 (2003).

[6] Dunn, R. C., "Near-Field Scanning Optical Microscopy", Chem. Rev. 99, 2891-2927 (1999).

[7] Hentschel, M., Schäferling, M., Metzger, B. and Giessen, H., "Plasmonic Diastereomers: Adding up Chiral Centers“, Nano Lett. 13(2), 600-606 (2013).

[8] Dregely, D., Neubrech, F., Duan, H., Vogelgesang, R. and Giessen, H., "Vibrational near-field mapping of planar and buried three-dimensional plasmonic nanostructures", Nat. Comm. 4, 2237 (2013).

[9] Baida, F.I., Van Labeke, D. and Pagani, Y., "Body-of-revolution FDTD simulations of improved tip performance for scanning near-field optical microscopes", Opt. Comm. 225(4-6), 241-252 (2003).

[10] Son, T., Kochuveedu, S. T., Kim, D. H. and Kim, D., "Near-field analysis of CdSe quantum dot conjugated coreshell nanoparticle", Proc. SPIE 8954, Nanoscale Imaging, Sensing, and Actuation for Biomedical Applications XI, 89540V (2014).

[11] Hecht, B., Heinzelmann H. and Pohl, D. W., "Combined aperture SNOM/PSTM: best of both worlds?”, Ultramicroscopy 57, 228-234 (1995).

[12] Robinson, J. T., Preble, S. F. and Lipson, M., "Imaging highly confined modes in sub-micron scale silicon waveguides using Transmissionbased Near-field Scanning Optical Microscopy”, Opt. Exp. 14(22), 10588 (2006).

[13] Schmid, M., Andrae, P. and Manley, P., "Plasmonic and photonic scattering and near-fields of nanoparticles", Nanoscale Research Letters 9, 50 (2014).

[14] Courjon, D. and Bainier, C., "Near-field microscopy and near-field optics", Rep. Prog. Phys. 57, 989-1028 (1994).

[15] Schnell, M., Garcia-Etxarri, A., Huber A. J., Crozier, K., Aizpurua, J. and Hillenbrand, R., "Controlling the nearfield oscillations of loaded plasmonic nanoantennas", Nature Photonics 3, 287-291 (2009).

[16] Hecht, B., Bielefeldt, H., Inouye, Y, Pohl, D. W. and Novotny, L., "Facts and artifacts in near-field optical microscopy", J. Appl. Phys. 81, 2492 (1997).

[17] Wu, S., "Review of near-field optical microscopy", Front. Phys. China 3, 263-274 (2006).

[18] Barchiesi, D., Bergossi, O., Pieralli, C. and Spajer, M., "Reflection scanning near-field optical microscopy (R$\mathrm{SNOM}$ ) in constant height mode with a dielectric probe Image interpretation and resolution for high topographic variations", Ultramicroscopy 71 (1-4)361-370 (1998).

[19] Girard, C., "Near-fields in nanostructures", Rep. Prog. Phys. 68, 1883 (2005).

[20] Moreno, E., Erni, D., Hafner, C. and Vahldieck, R., "Multiple multipole methode with automatic multipole setting applied to the simulation of surface plasmons in metallic nanostructures", JOAS A 19(1), 101-111 (2002).

[21] Kern A. M. and Martin, O. J.F., "Surface integral formulation for 3D simulations of plasmonic and high permittivity nanostructures", JOSA A 26(4), 732-740 (2009).

[22] Jalali, T., "Manipulation of lens-shaped objects in various materials to enhance photonic nanojetz using MMP method", J. Opt. 16(3), 035705 (2014).

[23] Novotny, L., Pohl, D. W., and Hecht, B., "Scanning near-field optical probe with ultrasmall spot size", Optics Letters 20(9), 970-972 (1995).

[24] Novotny, L. and Hafner, C., "Light propagation in a cylindrical waveguide with a complex, metallic, dielectric function", Phys. Rev. E 50, 4094-4106 (1994).

[25] Schmid, M., Grandidier, J. and Atwater, H. A., "Scanning near-field optical microscopy on dense random assemblies of metal nanoparticles", Journal of Optics 15(12), 125001 (2013). 
[26] Maier, S. A., Kik, G. P., Atwater, H. A., Meltzer, S., Harel, E., Koel B. E. and Requicha, A. A. G., "Local detection of electromagnetic energy transport below the diffraction limit in metal nanoparticle plasmon waveguides", Nat. Mat. 2, 229-232 (2003).

[27] Maier, S. A., Kik, G. P. and Atwater, H. A., "Observation of coupled plasmon-polariton modes in Au nanoparticle chain waveguides of different length: Estimation of waveguide loss", Appl. Phys. Lett. 81(9), 1714-1716 (2002).

[28] Maier, S. A., Brongersma, M. L., Kik, G. P. and Atwater, H. A., "Observation of near-field coupling in metal nanoparticle chains using far-field”, Phys. Re. B. 65, 1934908 (2002).

[29] Gupta, R., Dyer, M. J. and Weimer, W. A., "Preparation and characterization of surface plasmon resonance tunable gold and silver films", Journal of Applied Physics 92, 5264-5271 (2002).

[30] Hell, S. W., Booth, M., Wilms, S., Schnetter, C. M., Krisch, A. K., Arndt-Jovin, D. J. and Jovin, M. T., “Thwophoton near- and far-field fluorescence microscopy with continuous-wave excitation", Opt. Lett. 23(15), 12381240(1998).

[31] Bouillard, G. J-S., Dickson, W., Wurtz, G. A. and Zayats, A. V., "Near-Field Hyperspectral Imaging", Chem. Ohys. Chem. 10.1002 (2014).

[32] Ye, F., Merlo, J. M., Burn M. J. and Naughton, M. J., "Optical and electrical mappings of surface plasmon cavity modes", Nanophotonics (2014). 